\title{
HEMÓCITOS FAGOCITÁRIOS EM LARVAS DE DIATRAEA SACCHARALIS (FABRICIUS) (LEPIDOPTERA, PYRALIDAE)
}

\author{
Angela M.F. Falleiros ${ }^{1}$ \\ Elisa A. Gregório ${ }^{2}$
}

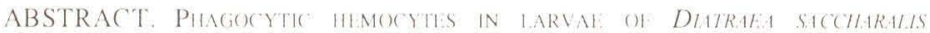
(FABRICHS) (LAPIDOPTIRA. PYRAIIDAE). The ultrastruetural ohservation of the hemocytes of $D$. sacharalis larvae showed different hemocy te types in various stage of interaction with microorganisms, which were interpreted as bacteria. The mieroorganisms were observed either free in the hemolymph or into the insect hemocyles. The bacteria were detected mostly into the granulocytes and plasmatocytes: the spherule cells and the oenocytoids also presented intracytoplasmic microorganisms. hut less frequently. The fagocylic capacity of the different hemocyte types is discussed.

KEY WORDS. Lepidoptera. Pyralidae. Diatraed saccharalis, hemocyte. phagocytosis. ultrastructure
\end{abstract}

As células sanguíneas dos insetos, os hemócitos, desempenham papéis de grande importância para a vida destes organismos, participando ativamente nos mecanismos de defesa contra elementos estranhos.

Os mecanismos de defesa dos insetos contra patógenos que penetram sua hemocele tem sido considerados como parte de um "sistema imune", muito embora não tenham sidos relacionados, até o presente, com a produção de imunoglobulinas (GUPTA 1985; RatclifFe et al. 1985).

Esses mecanismos de defesa podem ser do tipo celular e humoral. As reaçoes celulares, envolvendo diretamente os hemócitos, compreendem a coagulação da hemolinfa, a fagocitose, a formação de nódulos e o encapsulamento (GRIGOIRE \& GOFFINET 1979; LACKIE 1983; YAGER \& KNIGHT 1983; RIZKI \& RIZKI 1984; RATClifFe et al. 1985; GOTZ 1986). As reaçōes humorais, com o envolvimento indireto dos hemócitos, caracterizam-se pelo aparecimento na hemolinfa de fatores naturais ou induzidos tais como: aglutininas, fatores líticos, antibacterianos, lisosimas e a ativação do sistema de profenoloxidase (HOFFMANN et al. 1970; BeAllaton \& Monplyssin 1977; PENDland et al. 1988; BroOKMAN et al. 1989; DUNPHY \& CHADWICK 1989), entre outros.

1) Departanento de Histologia, Centro de Ciências Biológieas, Universidade Estadual de Londrina. Caixa Postal $(x)(0) 1$. $86(0) 51-970)$ Londrina. Paraná. Brasil.

2) Departamento de Morfologia. Instituto de Bociencias. Universidade Estadual Paulista. Caixa Postal 501. 18618-()(x) Botucatu. São Paulo). Brasil. 
A larva da Diatraea saccharalis (Fabricius, 1794), conhecida como broca da cana-de-açúcar, é a principal praga da cultura da cana-de-açucar em nosso país. Seis tipos de hemócitos foram identificados em sua hemolinfa por BOMBONATO (1994), a saber: prohemócito, plasmatócito, granulócito, esferulócito, oenocitóide e célula vermiforme. Os mecanismos de defesa deste inseto e suas relações com os hemócitos, entretanto, não foram estudados até a presente data.

Neste trabalho, apresenta-se achado ocasional de fagocitose e destruição de patógenos, por diferentes tipos de hemócitos em larvas de D. saccharalis.

\section{MATERIAL E MÉTODOS}

Os exemplares de larvas de $D$. saccharalis utilizados em nosso trabalho foram obtidos do Laboratório de Controle de Pragas da Usina da Barra Açúcar e Álcool S.A., Barra Bonita (São Paulo).

As larvas da $D$. saccharalis foram cultivadas em tubos de vidro previamente esterilizados, contendo dieta artificial desenvolvida por HENSLEY \& HAMMOND (1968), mantidas em temperatura de $25-27^{\circ} \mathrm{C}$ e umidade relativa do ar de aproximadamente $70 \%$.

A hemolinfa de 20 larvas com 24 dias de desenvolvimento foi coletada com auxílio de pipeta Pasteur, através de punção na região abdominal, imediatamente colocada em solução anti-coagulante de insetos (LEONARD et al. 1985) e centrifugada a $5000 \mathrm{rpm}$ por cinco minutos. O sedimento obtido foi fixado por duas horas em glutaraldeído a 2,5\% em tampão fosfato $0,1 \mathrm{M} \mathrm{pH} \mathrm{7,2} \mathrm{e} \mathrm{pós-fixado} \mathrm{em} \mathrm{tetróxido}$ de ósmio a $1 \%$ no mesmo tampão, sendo posteriormente processado rotineiramente para microscopia eletrônica de transmissão.

\section{RESULTADOS}

A análise morfológica ultraestrutural dos hemócitos de D. saccharalis mostrou, em algumas das preparações, microorganismos que foram interpretados como bactérias, livres ou em vários estágios de interação com diferentes tipos de hemócitos circulantes. As larvas infectadas não apresentavam qualquer alteração anatômica ou comportamental que as distinguissem das não contaminadas.

Os microorganismos foram observados preferencialmente nos granulócitos, que mostraram vacúolos em diversos estágios de digestão intracelular (Figs 1-4). Notamos adesão de grânulos citoplasmáticos, próprios deste tipo celular, ao vacúolo digestivo (Fig. 3), bem como vários patógenos destruídos (Fig. 4).

Imagens de fagocitose das bactérias pelos plasmatócitos foram observadas (Fig. 6); os patógenos intracelulares não estavam alterados, nestas células (Fig. 5).

Os oenocitóides (Fig. 7) e os esferulócitos (Fig. 8) também apresentaram microorganismos intracitoplasmáticos, embora com menor frequência. Nessas células, entretanto, nem sempre foi possível visualizar membrana vacuolar nítida, separando o citoplasma celular do patógeno, que se apresentava íntegro. 

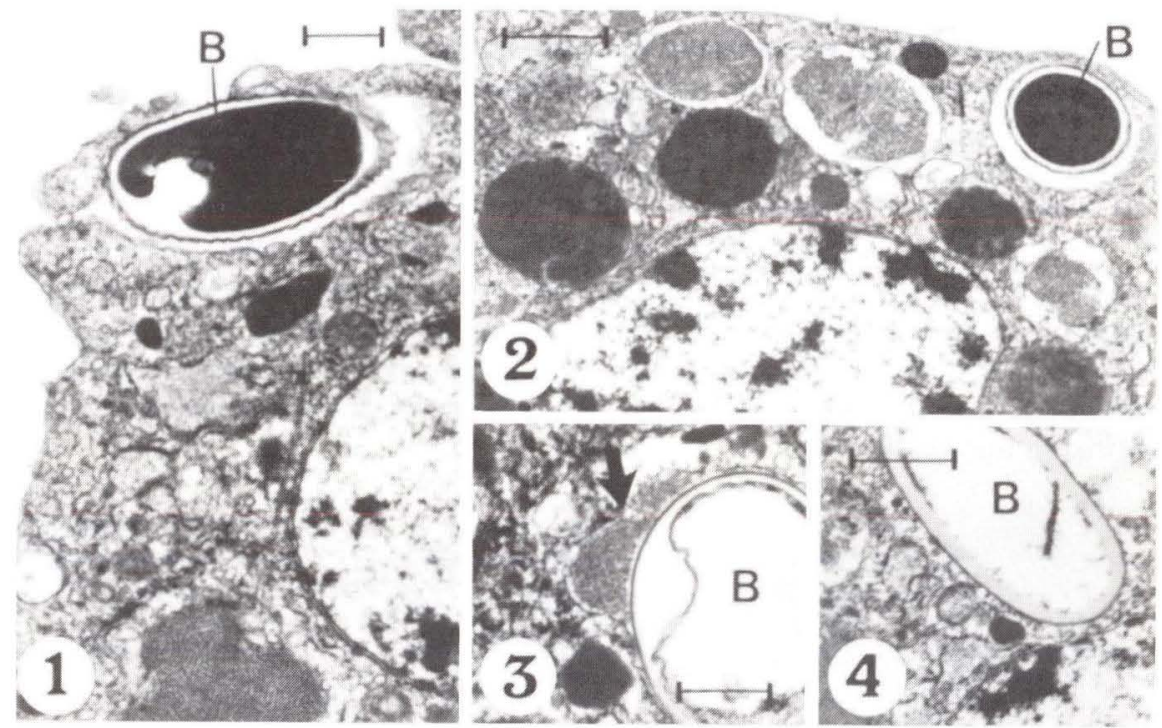

Figs 1-4. Granulócitos em diferentes fases da fagocitose de bactérias (B). (1) Emissão de pseudópodos (barra =0,5 $\mu \mathrm{m}$ ); (2) bactéria íntegra em vacúolo (barra $=1,0 \mu \mathrm{m}$ ); (3) detalhe de bactéria morta em vacúolo, notar imagem de fusão de grânulo com membrana vacuolar (seta) (barra $=0,5 \mu \mathrm{m})$; (4) bactéria morta (barra $=1,0 \mu \mathrm{m})$.

\section{DISCUSSÃO}

A fagocitose, considerada como um complexo e sofisticado processo biológico, é o mais comum dos mecanismos de imunidade celular, formando a primeira linha de defesa em muitos insetos (RIZKI \& RIZKI 1984; RATCLIFFE \& Rowley 1979; RATClifFe et al. 1985; ANDERSON \& CHAIN 1987; FENOGLIO et al. 1993).

Um grande número de microorganismos como vírus, fungos, bactérias e protozoários são fagocitados pelos hemócitos, tanto in vivo como in vitro (RATCLIFFE \& ROWLEY 1975 1979; RATCLIFFE \& WALTERS 1983; ChIANG et al. 1988; RAHMET-ALLA \& RowLEY 1990, entre outros). Os plasmatócitos (PL) e/ou granulócitos (GR) são os hemócitos apontados como as células fagocitárias, na grande maioria dos insetos (RowLEY \& RATCLIFFE 1976; RIZKI \& RIZKI 1984; GUPTA 1985; KAAYA et al. 1986; SANTOS et al. 1990; BAINES et al. 1992, EHLERS et al. 1992; HUNG et al. 1993), por possuirem a capacidade de reconhecer, aderir e englobar elementos estranhos ao organismo (RATCLIFFE \& ROWLEY 1979).

Alguns trabalhos mostraram que os PL são as células predominantemente envolvidas no processo de fagocitose (RIZKI \& RIZKI 1984; KAAYA et al. 1986; SANTOS et al. 1990; BAINES et al. 1992), enquanto outros autores descrevem como células fagocíticas predominantes os GR, (ROWLEY \& RATCLIFFE 1976; EHLERS et al. 1992; KURIHARA et al. 1992; Hung et al. 1993). Entretanto, ANGGRAENI 
\& RATCLIFFE (1991), trabalhando com monocamada de hemócitos purificados de Galleria mellonella (Linnaeus, 1758) (Lepidoptera, Pyralidae) coberta por suspensão bacteriana, observaram que, embora a ingestão fosse atributo dos PL, a atividade fagocitária máxima, nessas células, só foi obtida após a readição de GR ao meio, ilustrando a necessidade da cooperação célula-célula neste processo.

Outros tipos de hemócitos também foram apontados como fagócitos em insetos. NEUWIRTH (1974), trabalhando com inoculação de partículas inertes em larvas de Calpodes ethlius (Stoll, 1782) (Lepidoptera, Pyralidae), conclue que os GR e os oenocitóides (OE) são as células fagocíticas; os PL e os esferulócitos (ES) não fagocitam, embora partículas possam ser encontradas nas proximidades do primeiro tipo celular.
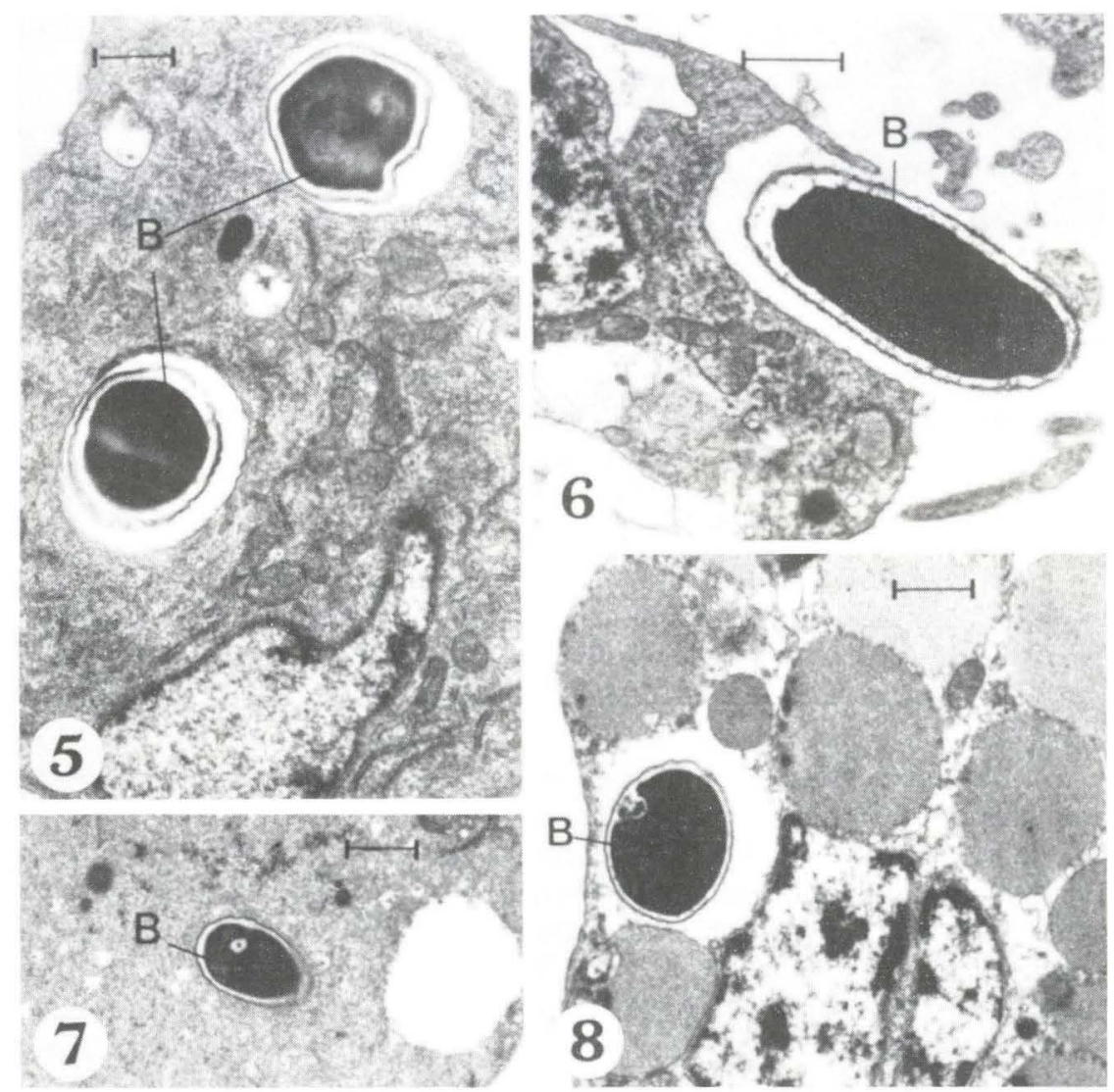

Figs 5-8. Diferentes tipos de hemócitos com bactéria (B) intracitoplasmática. (5) Plasmatócito contendo bactérias íntegras em vacúolo definido (barra $=0,5 \mu \mathrm{m}$ ); (6) plasmatócito englobando bactéria livre (barra $=1,0 \mu \mathrm{m})$; (7) oenocitóide (barra $=1,0 \mu \mathrm{m})$; $(8)$ esferulócito (barra $=1,0 \mu \mathrm{m})$. 
Em hemolinfa de larvas de $D$. saccharalis com 24 dias de desenvolvimento, as observações mostram que além dos PL e dos GR, também os ES e os OE se encontram, aparentemente, envolvidos na fagocitose de patógenos.

Estudos in vivo e in vitro sugerem que a fagocitose em insetos, a exemplo dos vertebrados, ocorre em quatro estágios: quimiotaxia, aderência, ingestão e morte do agente estranho (RATCLIFFE \& RowLEY 1979: RATClifFE 1986). Nas observações nota-se imagens que apontam para o GR e PL como células fagocitárias: bactérias foram vistas aderidas, sendo envoltas por pseudópodos e íntegras em vacúolos intracitoplasmáticos. Entretanto, somente o GR evidenciou bactérias em vários graus de degeneração, dentro dos vacúolos digestivos.

A habilidade de destruir os patógenos parece estar na dependéncia da liberação de enzimas lisosomais para os vacúolos de fagocitose (RATCLIFFE \& Rowley 1979; KROSCHINSKI \& RENWRANTZ 1988; Fenoglio et al. 1993). Nesse sentido, estudos citoquímicos ultraestruturais de detecção de atividade lisosomal deverão ser efetuados nos diferentes tipos de hemócitos, para comprovar com certeza aqueles que detém essa capacidade de destruição direta de patógenos.

Com relação aos ES e OE, pode-se observar bactérias no citoplasma, porém nem sempre com membrana vacuolar evidente. Segundo GuPTA (1985), estes últimos tipos celulares podem, ocasionalmente, serem apontados como fagócitos. porém, este fato não é demonstrado pela grande maioria dos autores. A hipótese de uma super infecçào bacteriana, que levasse ao comprometimento passivo de todas os tipos de hemócitos, inclusive ES e OE, pode ser descartada, uma vez que não se pôde distinguir, pelo aspecto macroscópico das larvas, aquelas que estavam contaminadas.

Não se conseguiu detectar bactérias no citoplasma de células vermiformes ou de prohemócitos. Esses tipos celulares aparecem em quantidade reduzida na hemolinfa da broca da cana com 24 dias de desenvolvimento (BOMBONATO 1994). Assim, a dificuldade natural de detetá-los nas preparaçōes para microscópio eletronico, não permite excluí-tos da relação dos hemócitos fagocitários em $D$. saccharalis.

A capacidade fagocitária dos diferentes tipos de hemócitos da $D$. sacharalis deverá ser experimentalmente avaliada, bem como o papel de cada um deles nos mecanismos de defesa deste inseto.

AGRADECIMENTOS, Os autores agradecen as Sras. Marra Euleda Lino Perez e Maria Helena Moreno, pelo processamento técnico do material. Trabalho parcialmente financiado pelo CNPQ (Proc. 140179/9()-9)

\section{REFERÊNCIAS BIBLIOGRÁFICAS}

ANderson, R. \& B.M. ChaIN. 1987. Macrophage functions in insects, p.77-88. In: A.P. GuPTA (Ed.). Hemocytic and humoral immunity in arthropods. New York, Willey-Interscience, XII +533p.

ANGgraeni, T. \& N.A. RatClifFE. 1991. Studies on cell-cell co-operation during 
phagocytosis by purified haemocyte populations of the moth, Galleria mellonella. Jour. Insect Physiol. 37 (6): 453-460.

Baines, D.; T. DeSAntis \& R.G.H. Downer. 1992. Octopamine and 5-hydroxytryptamine enchance the phagocytic and nodule formation activities of cockroach (Periplaneta americana) haemocytes. Jour. Insect Physiol. 38 (11): 905-914.

Beaulaton, J. \& M. Monpeyssin. 1977. Ultrastructure et cytochimie des hémocytes d'Antheraea pernyi (Guér) (Lepidoptera, Attacidae). II Cellules à sphèrules et oenocytoides. Biol. Cell. 28: 13-18.

Bombonato, M.T.S. 1994. Análise morfológica e quantitativa dos hemócitos de larvas de Diatraea saccharalis (Lepidoptera: Pyralidae) frente ao parasitóide Metagonistylum minense (Diptera: Tachinidae). Dissertação de Mestrado, não publicada, Instituto de Biociências, Universidade Estadual Paulista, Botucatu, 90p.

Brookman, J.L.; N.A. Ratcliffe \& A.F. Rowley. 1989. Studies on the activation of the prophenoloxidase system of insects by bacterial cell wall components. Insect Biochem. 19: 47-57.

ChiAnG, A.S.; A.P. Gupta \& S.S Han. 1988. Arthropod immune system: I. Comparative ligth and electron microscopic accounts of immunocytes and other hemocytes of Blattella germanica (Dictyoptera: Blattellidae). Jour. Morphol. 198: $257-267$.

Dunphy, G.B. \& J.S. CHADWICK. 1989. Effects of selected carbohydrate and the contribution of the prophenoloxidase cascade system to the adhesion of strains of Pseudomonas aeruginosa and Proteus mirabilis to hemocytes of nonimmune larval Galleria mellonella. Can. Jour. Microbiol. 35: 524-527.

Ehlers, D.; B. Zosel; W. Mohrig; E. Kauschke \& M. Ehlers. 1992. Comparison of in vivo and in vitro phagocytosis in Galleria mellonella L.. Parasitol. Res. 78: 354-359.

Fenoglio, C.; P. Bernardini \& M.V. Gervaso. 1993. Cytochemical characterization of the hemocytes of Leucophaea maderae (Dictyoptera: Blaberoidea). Jour. Morphol. 218: 115-126.

GöTz, P. 1986. Mechanisms of encapsulation in Diptera hosts, p. 1-19. In: A.M. LACKIE (Ed.). Immune mechanisms in invertebrate vectors. Oxford, Claredon Press, $\mathrm{V}+285 \mathrm{p}$.

Grégorre, C.H. \& G. GofFinet. 1979. Controversies about the coagulocytes, p. 189-229. In: A.P. GuPTA (Ed.). Insect hemocytes: development, forms, functions and techniques. Cambridge, Cambridge University Press, XVI+ $614 \mathrm{p}$.

GuPTA, A.P. 1985. Cellular elements in the hemolymph, p.402-444. In: G.A. KERKUT \& L.I. GILBERT (Eds). Comprehensive insect physiology biochemistry and pharmacology. Oxford, Pergamon Press, vol. 3, XVI+625p.

HENSLEY, S.D. \& A.M. HAMMOND JR. 1968. Laboratory techniques for rearing the sugarcane borer on an artificial diet. Jour. Econ. Entomol 61: 1742-1743. Hoffmann, J.A.; A. PORTE \& P. Joly. 1970. On the localization of phenoloxi- 
dase activity in coagulation of Locusta migratoria (L.) (Orthoptera). C.R. Hebd. Séances Acad. Sc. Paris. 270: 629-631.

HunG, S. Y.; D.G. Boucias \& A.J. VEY. 1993. Effect of Beauveria bassiana and Candida albicans on the cellular defense response of Spodoptera exigua. Jour. Invertebr. Pathol. 61: 179-187.

KaAya, G.P.; N.A. Ratcliffe \& P. Alemu. 1986. Cellular and humoral defenses of Glossina: reactions against bacteria, trypanosomes and experimental implants. Jour. Med. Entomol. 23: 30-43.

Krochinski, J. \& L. Renwrantz. 1988. Determination of the $\mathrm{pH}$ values inside the digestive vacuoles of hemocytes from Mytilus edulis. Jour. Invertebr. Pathol. 51: 73-79.

Kurihara, Y.; T. Shimazu \& H. Wago. 1992. Classification of hemocytes in the common cutworm, Spodoptera litura (Lepidoptera: Noctuidae) I. Phase microscopic study. Appl. Entomol. Zool. 27 (2): 225-235.

LACKIE, A.M. 1983. Effects of substratum wettability and charge on adhesion in vitro and encapsulation in vivo by insect haemocytes. Jour. Cell Sci. 63: 181-190.

Leonard, C.; K. Soderhall \& N.A. Ratcliffe. 1985. Studies of pro-phenoloxidase and protease of Blaberus craniifer haemocytes. Insect Biochem. 15: 803-810.

NeuwirTh, M. 1974. Granular hemocytes, the main phagocytic blood cells in Calpodes ethlius (Lepidoptera, Hesperiidae). Can. Jour. Zool. 52: 783-784.

Pendland, J.C.; M.A. Heat \& D.G. Boucias. 1988. Functions of a galactosebinding lectin from Spodoptera exigua larval haemolymph: opsonization of blastospores from entomogeneous hyphomycetes. Jour. Insect Physiol. 34: 533-540.

Rahmet-Alla, M. \& A.F. Rowl.Fy. 1990. Studies on the cellular defense reactions of the madeira cockroach, Lencophaea maderae: in vitro phagocytosis of different strains of Bacillus cereus and their effect on hemocyte viability. Jour. Invertebr. Pathol. 55: 350-356.

RATCLIFFE, N.A. 1986. Insect cellular immunity and the recognition of foreignness, p.21-43. In: A.M. LACKIE (Ed.). Immune mechanisms in invertebrate vectors. Oxford, Claredon Press, V $+285 p$.

RatclifFe, N.A. \& A.F. Rowley. 1975. Cellular defense reactions of insect hemocytes in vitro: phagocytosis in a new suspension culture system. Jour. Invertebr. Pathol. 26: 225-233.

1979. Role of hemocytes in defense against biological agents, p.331-414. In: A.P. GUPTA (Ed.). Insect hemocytes: development, forms, functions and techniques. Cambridge, Cambridge University Press. XVI $+614 p$.

Ratcliffe, N.A. \& J.B. Walters. 1983. Studies on the in vivo cellular reactions of insects: clearance of pathogenic and non-pathogenic bacteria in Galleria mellonella larvae. Jour. Insect Physiol. 29: 407-415.

Ratcliffe, N.A.; A.F. Rowley; S.W. Fitzgerald \& C.P. Rhodes. 1985. Invertebrate immunity: basic concepts and recent advances. Int. Rev. Cytol. 
97: $183-349$.

Rizkı, T.M. \& R.M. RIzKI. 1984. The cellular defense of Drosophila melanogaster, p.579-604. In: R.C. KING \& H. AKAI (Eds). Insect Ultrastructure. New York, Plenum Press, vol. 2, XXV +624p.

Rowley, A.F. \& N.A. RATClifFE. 1976. The granular cells of Galleria mellonella during clotting phagocytic reactions in vitro. Tissue Cell. 8 (3): 437-446.

Santos, R.R.; M.M.G. Este; E.S. Garcia \& P. Azambuja. 1990. Binding sites for $\operatorname{IgG}-\mathrm{Fc}$ in hemocyte adherent cells of hematophagous bugs (Rhodnius prolixus). Mem. Inst. Oswaldo Cruz 85 (4): 469-470.

YAGER, J.F. \& H.H. KNIGHT. 1983. Microscopic observation blood coagulation in several differents species of insects. Jour. Ent. Soc. Amer. 26: 591-602. 